\title{
Calambres musculares en los miembros inferiores (Observación preliminar: efecto beneficioso de la Trimetazidina)
}

\author{
José Luis del Burgo Fernándeza
}

\begin{abstract}
a Médico de Familia.
Centro de Salud de Porzuna (Ciudad Real).

Correspondencia:

José Luis del Burgo

Fernández. Centro de Salud de Porzuna, Av. del Dr. Garrido s/n, 13120 - Porzuna

(Ciudad Real).

E-mail: jldelburgo@majada.net
\end{abstract}

Recibido el 28 de agosto de 2008.

Aceptado para su publicación el 10 de septiembre de 2008.

\section{RESUMEN}

Se expone una serie de observaciones relacionadas con la desaparición o mejoría de los calambres nocturnos en los miembros inferiores durante la utilización de trimetazidina con otros fines terapéuticos.

A partir del efecto beneficioso sobre los calambres musculares nocturnos en una paciente en tratamiento con trimetazidina, se realiza una búsqueda bibliográfica sin obtenerse ningún resultado. Se identifican pacientes en tratamiento con trimetazidina que refieran presentar o haber presentado episodios de calambres. Se localiza un total de 7 pacientes, de los cuales 6 refieren una franca mejoría de los calambres musculares desde el inicio del tratamiento con trimetazidina, sobre todo en lo referente a intensidad y frecuencia de los mismos.

Los efectos observados sugieren un efecto beneficioso de la trimetazidina en los calambres musculares y por tanto un potencial uso terapéutico en los mismos.

Palabras clave. Calambre Muscular, Extremidad Inferior, Trimetazidina.

\section{ABSTRACT}

Leg cramps (Preliminary observation: beneficial effect of Trimetazidine)

Leg cramps are a common condition for which the treatment of reference, quinine, can cause frequent and potentially serious side effects. Here, we describe a series of observations related to the disappearance or improvement of night-time leg cramps during treatment with trimetazidine used for other therapeutic purposes.

Starting from the hypothesis of trimetazidine treatment possibly having a beneficial effect on nocturnal muscle cramps, a literature search was carried out but with no significant results. Patients in treatment with trimetazidine referring to presenting or having presented episodes of cramps were identified. These corresponded to a total of 7 patients in whom 6 referred to a frank improvement of the muscle cramps from the beginning of treatment with trimetazidine, mainly in relation to the intensity and frequency of the cramps.

The observed effects suggest a beneficial effect of trimetazidine onmuscle cramps and, therefore, its potential therapeutic use for this indication.

Key words. Muscle Cramp, Lower Extremity, Trimetazidine.

\section{INTRODUCCIÓN}

Los calambres en los miembros inferiores son una patología frecuente que, si bien rara vez es grave, puede suponer para los pacientes que los sufren una seria amenaza para el descanso nocturno y su calidad de vida, y en ocasiones también para el desarrollo de actividades laborales y de ocio ${ }^{1,2}$.

Algunos tratamientos han mostrado un modesto efecto en pacientes con calambres asociados a cirrosis ${ }^{3}$ o gestación ${ }^{4}$, pero solo la quinina ha mostrado un efecto beneficioso frente a placebo ${ }^{5,6,7}$, aunque un metaanálisis, incluyendo ensayos clínicos no publicados, modera de forma notable el efecto beneficioso de este fármaco ${ }^{8}$, lo que unido a sus efectos secundarios potencialmente serios hace que algunos autores aconsejen reservarla para aquellos pacientes con formas especialmente serias e invalidantes de calambres ${ }^{2,6}$.

La trimetazidina es un derivado piperazínico que se utiliza esencialmente en el tratamiento del vértigo periférico, los acúfenos, la hipoacusia perceptiva, retinopatías isquémicas y en la angina de esfuerzo $0^{9,10}$. Generalmente bien tolerada, en un estudio realizado en 149 pacientes con dosis doble de la habitual de trimetazidina se encontró un $29.6 \%$ 


\begin{tabular}{|c|c|c|c|c|c|c|c|}
\hline PACIENTE & 1 & 2 & 3 & 4 & 5 & 6 & 7 \\
\hline EDAD & 62 & 78 & 78 & 81 & 78 & 60 & 60 \\
\hline SEXO & $\mathrm{F}$ & $M$ & $\mathrm{~F}$ & $\mathrm{~F}$ & M & $\mathrm{M}$ & $\mathrm{F}$ \\
\hline $\begin{array}{l}\text { PATOLOGIA ASO- } \\
\text { CIADA }\end{array}$ & $\begin{array}{l}\text { Vértigo } \\
\text { HTA } \\
\text { Migraña } \\
\text { Hiperlipide- } \\
\text { mia mixta } \\
\text { Espondi- } \\
\text { loartrosis }\end{array}$ & $\begin{array}{l}\text { HTA } \\
\text { Diabetes } \\
\text { mellitus } \\
\text { AIT } \\
\text { Artrosis } \\
\text { EPOC } \\
\text { Insuf ve- } \\
\text { nosa }\end{array}$ & $\begin{array}{l}\text { HTA } \\
\text { Cardiopatía } \\
\text { isquémica } \\
\text { Hiperlipide- } \\
\text { mia mixta } \\
\text { Trombope- } \\
\text { nia } \\
\text { Valvulopatía } \\
\text { mitro-aor- } \\
\text { tica } \\
\text { Gammapa- } \\
\text { tia policlonal } \\
\text { Cataratas } \\
\text { Hiperamila- } \\
\text { semia }\end{array}$ & $\begin{array}{l}\text { HTA } \\
\text { Obesidad } \\
\text { Cardiopatía } \\
\text { HTA } \\
\text { Diabetes } \\
\text { mellitus } \\
\text { Fibrilación } \\
\text { auricular } \\
\text { Hipercoles- } \\
\text { terolemia } \\
\text { ACV }\end{array}$ & $\begin{array}{l}\text { HTA } \\
\text { Obesidad } \\
\text { Hipercoles- } \\
\text { terole } \\
\text { mia } \\
\text { Poliartrosis } \\
\text { Trast ansio- } \\
\text { so-depre- } \\
\text { sivo } \\
\text { Glucemia } \\
\text { basal alte- } \\
\text { rada }\end{array}$ & $\begin{array}{l}\text { Cirrosis } \\
\text { hepática no } \\
\text { alcoholica } \\
\text { Hiperten- } \\
\text { sión portal } \\
\text { Hiperurice- } \\
\text { mia } \\
\text { Hernia } \\
\text { inguinal } \\
\text { Hiperlipide- } \\
\text { mia mixta }\end{array}$ & $\begin{array}{l}\text { Hernia de } \\
\text { hiato } \\
\text { Hiperpara- } \\
\text { tiroi- } \\
\text { dismo } \\
\text { Enf. de } \\
\text { Chron } \\
\text { Artrosis } \\
\text { Osteopenia } \\
\text { Osteoma } \\
\text { osteoide } \\
\text { Acúfenos } \\
\text { Despren- } \\
\text { dimiento } \\
\text { vítreo } \\
\text { Disquinesia } \\
\text { biliar }\end{array}$ \\
\hline $\begin{array}{l}\text { TRATAMIENTOS } \\
\text { ACTUALES }\end{array}$ & $\begin{array}{l}\text { Valsartan } \\
\text { Hidrocloro- } \\
\text { tiazida } \\
\text { Rizatriptan } \\
\text { Simvasta- } \\
\text { tina }\end{array}$ & $\begin{array}{l}\text { Insulina } \\
\text { Valsartan } \\
\text { Hidrocloro- } \\
\text { tiazida } \\
\text { Atorvasta- } \\
\text { tina } \\
\text { Ezetimiba } \\
\text { Nabume- } \\
\text { tona } \\
\text { Paracetamol } \\
\text { AAS } \\
\text { Pregabalina } \\
\text { Clopidogrel }\end{array}$ & $\begin{array}{l}\text { AAS } \\
\text { Ramipril } \\
\text { Dinitrato de } \\
\text { isosorbide } \\
\text { Bisoprolol } \\
\text { Amlodipino } \\
\text { Torasemida } \\
\text { Simvasta- } \\
\text { tina } \\
\text { Omeprazol } \\
\text { Paracetamol }\end{array}$ & $\begin{array}{l}\text { Nifedipino } \\
\text { Doxazosina } \\
\text { Eprosartan } \\
\text { Hidrocloro- } \\
\text { tiazida } \\
\text { Digoxina } \\
\text { Furosemida } \\
\text { Metformina } \\
\text { Glimepirida } \\
\text { Ezetimiba } \\
\text { Pantoprazol } \\
\text { Acenocu- } \\
\text { marol }\end{array}$ & $\begin{array}{l}\text { Paracetamol } \\
\text { Tramadol } \\
\text { Ezetimiba } \\
\text { Omeprazol } \\
\text { Paroxetina } \\
\text { Lorazepam } \\
\text { Indapamida }\end{array}$ & $\begin{array}{l}\text { Norfloxa- } \\
\text { cino } \\
\text { Torasemida } \\
\text { Espironola- } \\
\text { ctona } \\
\text { Alopurinol } \\
\text { Omeprazol } \\
\text { Loracepan } \\
\text { Paracetamol }\end{array}$ & $\begin{array}{l}\text { Azathiprina } \\
\text { Budesonida } \\
\text { Omeprazol } \\
\text { Lactitol } \\
\text { Acido fólico } \\
\text { Raloxifeno } \\
\text { Paracetamol }\end{array}$ \\
\hline $\begin{array}{l}\text { ANTECEDENTES } \\
\text { FAMILIARES }\end{array}$ & No & No & No & No & No & Sí (Padre) & No \\
\hline $\begin{array}{l}\text { FARMACOS PO- } \\
\text { TENCIALMENTE } \\
\text { IMPLICADOS }\end{array}$ & $\begin{array}{l}\text { Simvasta- } \\
\text { tina } \\
\text { Hidrocloro- } \\
\text { tiazida }\end{array}$ & $\begin{array}{l}\text { Atorvasta- } \\
\text { tina } \\
\text { Hidrocloro- } \\
\text { tiazida }\end{array}$ & $\begin{array}{l}\text { Amlodipino } \\
\text { Torasemida } \\
\text { Sivastatina } \\
\text { Ramipril } \\
\text { Bisoprolol }\end{array}$ & $\begin{array}{l}\text { Hidrocloro- } \\
\text { tiazida } \\
\text { Furosemida } \\
\text { Nifedipino }\end{array}$ & Indapamida & $\begin{array}{l}\text { Torasemida } \\
\text { Espironola- } \\
\text { ctona }\end{array}$ & Raloxifeno \\
\hline $\begin{array}{l}\text { PATOLOGIA } \\
\text { POTENCIALMEN- } \\
\text { TE IMPLICADA } \\
\left(\text { CLAMBRES } 2^{\circ}\right)\end{array}$ & No & $\begin{array}{l}\text { Diabetes } \\
\text { mellitus } \\
\text { Insuf. Ve- } \\
\text { nosa }\end{array}$ & No & $\begin{array}{l}\text { Diabetes } \\
\text { mellitus }\end{array}$ & No & $\begin{array}{l}\text { Cirrosis } \\
\text { hepática }\end{array}$ & $\begin{array}{l}\text { Hiperparati- } \\
\text { roi-dismo }\end{array}$ \\
\hline $\begin{array}{l}\text { ANOMALIAS } \\
\text { EXPLORATORIAS } \\
\text { (pulsos pedios, } \\
\text { tibiales, bocio, } \\
\text { fasciculaciones, } \\
\text { alteraciones mus- } \\
\text { culares, deshidra- } \\
\text { tación,.. ) }\end{array}$ & No & $\begin{array}{l}\text { Pedios y } \\
\text { tibiales } \\
\text { atenuados } \\
\text { ROT ate- } \\
\text { nuados. }\end{array}$ & No & $\begin{array}{l}\text { Tibiales } \\
\text { atenuados }\end{array}$ & No & No & No \\
\hline $\begin{array}{l}\text { ANOMALIAS } \\
\text { ANALITICAS } \\
\text { (iones, creatinina, } \\
\text { transaminasas, } \\
\text { ALP, CPK, TSH) }\end{array}$ & No & $\begin{array}{l}\text { Glucemia } \\
203\end{array}$ & $\begin{array}{l}\text { Creatinina } \\
1.3\end{array}$ & $\begin{array}{l}\text { Glucemia } \\
179\end{array}$ & No & $\begin{array}{l}\text { GOT } 43 \\
\text { GGT } 51\end{array}$ & PTH 59.6 \\
\hline $\begin{array}{l}\text { ORIENTACION } \\
\text { DIAGNOSTICA } \\
\text { DE LOS CALAM- } \\
\text { BRES }\end{array}$ & $\begin{array}{l}\text { Calambres } \\
\text { nocturnos } \\
\text { idiopáticos. }\end{array}$ & $\begin{array}{l}\text { Calambres } \\
\text { nocturnos } \\
\text { idiopáticos }\end{array}$ & $\begin{array}{l}\text { Calambres } \\
\text { nocturnos } \\
\text { idiopáticos }\end{array}$ & $\begin{array}{l}\text { Calambres } \\
\text { nocturnos } \\
\text { idiopáticos }\end{array}$ & $\begin{array}{l}\text { Calambres } \\
\text { nocturnos } \\
\text { idiopáticos }\end{array}$ & $\begin{array}{l}\text { Calambres } \\
\text { de etiología } \\
\text { posiblemen- } \\
\text { te mixta }\end{array}$ & $\begin{array}{l}\text { Calambres } \\
\text { de etiología } \\
\text { potencial- } \\
\text { mente mixta }\end{array}$ \\
\hline
\end{tabular}

Tabla 1. Características clínicas de los pacientes en tratamiento con trimetazidina y presencia de calambres musculares. 


\begin{tabular}{|c|c|c|c|c|c|c|c|}
\hline PACIENTE & 1 & 2 & 3 & 4 & 5 & 6 & 7 \\
\hline $\begin{array}{l}\text { AÑOS EVOLU- } \\
\text { CION }\end{array}$ & $1-2$ & 30 & $6-7$ & 2 & $3-4$ & 0,5 & Años (¿?) \\
\hline \multirow[t]{2}{*}{$\begin{array}{l}\text { LOCALIZA- } \\
\text { CION }\end{array}$} & $\begin{array}{l}\text { Pantorri- } \\
\text { llas }\end{array}$ & Pantorrilla & $\begin{array}{c}\text { Ambas } \\
\text { pantorrillas }\end{array}$ & $\begin{array}{c}\text { Ambas } \\
\text { pantorrillas }\end{array}$ & Pantorrilla & $\begin{array}{l}\text { Dorso de los } \\
\text { pies }\end{array}$ & $\begin{array}{c}\text { Ambas panto- } \\
\text { rrillas / ambos } \\
\text { pies }\end{array}$ \\
\hline & $\begin{array}{l}\text { Pantorri- } \\
\text { llas }\end{array}$ & Pantorrilla & $\begin{array}{c}\text { Ambas } \\
\text { pantorrillas }\end{array}$ & $\begin{array}{l}\text { No Calam- } \\
\text { bres }\end{array}$ & $\begin{array}{l}\text { No calam- } \\
\text { bres }\end{array}$ & No calambres & $\begin{array}{c}\text { Ambas panto- } \\
\text { rrillas / ambos } \\
\text { pies }\end{array}$ \\
\hline \multirow[t]{2}{*}{$\begin{array}{l}\text { MOMENTO } \\
\text { DE APARI- } \\
\text { CIÓN }\end{array}$} & $\begin{array}{l}\text { Durante } \\
\text { el sueño }\end{array}$ & $\begin{array}{l}\text { Al levan- } \\
\text { tarse }\end{array}$ & $\begin{array}{l}\text { Durante el } \\
\text { sueño }\end{array}$ & $\begin{array}{l}\text { Previo } \\
\text { sueño }\end{array}$ & $\begin{array}{l}\text { Al Levan- } \\
\text { tarse }\end{array}$ & $\begin{array}{l}\text { Previo al sue- } \\
\text { ño/ Durante el } \\
\text { sueño }\end{array}$ & $\begin{array}{c}\text { Durante el } \\
\text { sueño / Con el } \\
\text { ejercicio }\end{array}$ \\
\hline & $\begin{array}{l}\text { Durante } \\
\text { el sueño }\end{array}$ & $\begin{array}{l}\text { Al levan- } \\
\text { tarse }\end{array}$ & $\begin{array}{l}\text { Durante el } \\
\text { sueño }\end{array}$ & $\begin{array}{l}\text { No calam- } \\
\text { bres }\end{array}$ & $\begin{array}{l}\text { No calam- } \\
\text { bres }\end{array}$ & No calambres & $\begin{array}{l}\text { Con el ejer- } \\
\text { cicio }\end{array}$ \\
\hline \multirow[t]{2}{*}{$\begin{array}{l}\text { DURACION } \\
\text { HABITUAL }\end{array}$} & $\begin{array}{l}5-10 \\
\min .\end{array}$ & $5-10 \mathrm{~min}$ & $<5 \min$ & $5-10 \mathrm{~min}$ & $>10 \mathrm{~min}$ & $5-10 \min$. & $5-10 \mathrm{~min}$ \\
\hline & $\begin{array}{l}\text { Segun- } \\
\text { dos }\end{array}$ & $5-10 \mathrm{~min}$ & $5-10 \mathrm{~min}$ & $\begin{array}{l}\text { No calam- } \\
\text { bres }\end{array}$ & $\begin{array}{l}\text { No calam- } \\
\text { bres }\end{array}$ & No calambres & $5-10 \min$ \\
\hline \multirow[t]{2}{*}{ FRECUENCIA } & Diaria & Casi diaria & Ocasional & Casi diaria & Casi diaria & Semanal & Casi diaria \\
\hline & Ocasional & Ocasional & Semanal & No & No & No & Ocasional \\
\hline \multirow{2}{*}{$\begin{array}{l}\text { INTENSIDAD } \\
\text { (E.V.A.) }\end{array}$} & 6.5 & 8 & 1 & 7 & 10 & 4.5 & 9 \\
\hline & 3.5 & 5 & 10 & 0 & 0 & 0 & 8 \\
\hline
\end{tabular}

Tabla 2. Evolución de los calambres musculares bajo el tratamiento con trimetazidina. A: antes del inicio del tratamiento con trimetazidina. D: después del inicio del tratamiento con trimetazidina. EVA: Escala visual analógica. Los valores de la EVA

con decimales se deben a la valoración realizada por el paciente del tipo "entre 4 y 9 ".

de efectos secundarios, destacando entre ellos la fatiga (7\%), el mareo (7\%), los calambres musculares $(7 \%)$ y el malestar inducido por el esfuerzo (5.6\%) ${ }^{11}$.

A continuación se expone una serie de observaciones relacionadas con la desaparición o mejoría de los calambres nocturnos en los miembros inferiores durante la utilización de trimetazidina con otros fines terapéuticos.

\section{OBSERVACIONES CLÍNICAS}

El caso índice lo constituye una paciente de 62 años de edad con antecedentes personales de herpes zoster, histerectomía a los 25 años de edad y artritis séptica fímica en rodilla izquierda a los 31 años, con anquilosis residual. Está diagnosticada de fascitis plantar, hipertensión arterial, vértigo multifactorial, migraña común, hiperlipidemia mixta y espondiloatrosis. Sigue tratamiento con valsartán e hidroclorotiazida, simvastatina y rizatriptam. Por acúfenos y sensación vertiginosa con inestabilidad se instauró tratamiento con trimetazidina a dosis de $20 \mathrm{mg}$ cada 8 horas. Ante la mala respuesta inicial se remitió a la paciente a la consulta de Otorrinolaringología para revisión, donde sustituyeron la trimetazidina por extracto de ginkgo biloba. Cuando acudió a revisión, la paciente refirió encontrarse igual de su cuadro vertiginoso, pero había empeorado de los calambres nocturnos que sufre en ambas piernas desde hace 2-3 años y que habían desaparecido con el uso de la trimetazidina. Ante esta afirmación se procedió a descartar causas secundarias de calambres musculares mediante anamnesis, exploración y revisión de los resultados de laboratorio, resultando ser un cuadro de calambres idiopáticos nocturnos.

Se realizó una búsqueda bibliográfica en Medline con los términos "muscle cramps AND trimetazidine" sin limitadores, encontrándose 0 resultados. La búsqueda en IBECS con el término "trimetazidina" produjo 7 entradas, ninguna de las cuales hacía referencia a calambres musculares. En la base de tesis doctorales TESEO se encontraron dos entradas con "trimetazidina" referentes al efecto citoprotector frente a la isquemia miocárdica en modelos experimentales. Por último, en IME la búsqueda con los parámetros "trimetazidina (titulo en español) Y calambres (título en español)" tampoco produjo ningún resultado y la sustitución del operador booleano $Y$ por $O$ produjo 22 entradas, ninguna de las cuales relacionaba la patología con el fármaco. Finalmente, una búsqueda libre en Google con los términos trimetazidina y calambres proporciona 


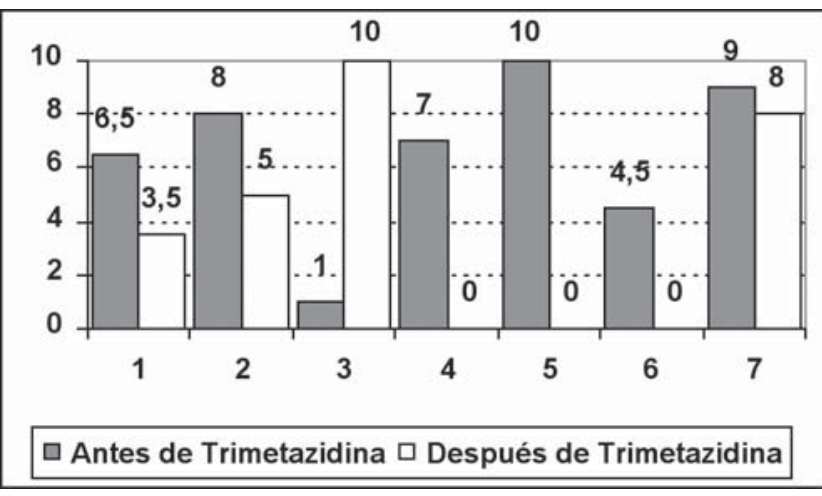

Figura 1. Intensidad de los calambres medido con EVA.

114 entradas, no habiéndose encontrado ninguna que relacione la trimetazidina como tratamiento de los calambres musculares.

También se realizó una búsqueda en la base de datos de historias clínicas del cupo médico al que pertenece la paciente de otros pacientes en tratamiento con trimetazidina a fin de localizar a aquéllos que además refieran presentar o haber presentado episodios de calambres. Se identificaron 6 pacientes más en los que, tomando trimetazidina, referían presentar o haber presentado calambres musculares en los miembros inferiores, procediéndose a su entrevista, exploración y revisión de historia clínica. Los resultados se exponen en las tablas 1 y 2

\section{DISCUSIÓN Y CONCLUSIONES}

El uso de la trimetazidina en estos pacientes ha supuesto una mejora en su situación general excepto en uno, sobre todo en la frecuencia de la aparición de los mismos, que pasa de ser predominantemente "casi diaria" a "ocasional" o "ausente". En cuanto a la intensidad dolorosa del calambre, también se aprecia una mejoría general, que en tres pacientes supone la ausencia de dolor (figura 1). La valoración de la duración de los mismos se hace mas difícil de realizar dada la variabilidad que se aprecia entre los 7 pacientes.

El único paciente que presenta un claro empeoramiento de los calambres se hallaba en tratamiento con trimetazidina desde antes de iniciarse la aparición de los mismos, por lo que tanto en frecuencia como en duración e intensidad los datos son negativos.

A pesar de lo reducido de la serie, estos datos podrían sugerir la hipótesis de un potencial efecto beneficioso de la trimetazidina en el tratamiento de los calambres en general, tanto en el calambre nocturno idiopático (con mucho el más frecuente de todos), como el asociado a algunas alteraciones somáticas (cirrosis, consumo de fármacos, etc.).
La forma de actuar de la trimetazidina como protector celular ${ }^{9,10,12}$, oponiéndose a los efectos nocivos de los radicales libres, evitando la caída del metabolismo energético durante la isquemia tisular, disminuyendo la acidosis intracelular y corrigiendo las alteraciones de los flujos iónicos transmembrana provocados por la isquemia, podrían estar en la base de la corrección de los calambres musculares.

La utilidad de este potencial efecto de la trimetazidina se derivaría de la alta prevalencia del calambre muscular como enfermedad en sí misma (prevalencia que aumenta con la edad, llegando a afectar a un tercio de la población mayor) y en los potencialmente serios secundarismos de la quinina como actual fármaco de referencia.

No obstante y dada la naturaleza del origen de los datos, se hace necesario profundizar en el estudio de la posible aplicación terapéutica de la trimetazidina en los calambres musculares de diferente origen, especialmente a través de ensayos clínicos específicos bien diseñados.

\section{BIBLIOGRAFÍA}

1. Millar TM, Layzer RB. Muscle cramps. Muscle Nerve 2005; 32(4):431-42.

2. Tranche Iparraguirre $S$. Calambres en las pantorrillas. AMF 2007; 3(8):460-64.

3. Kugelmas M. Preliminary observation: oral zinc sulfate replacement is effective in treating muscle cramps in cirrhotic patients. J Am Coll Nutr 2000; 19(1):13-5.

4. Roffe C, Sills S, Crome P, Jones P. Randomised, cross-over, placebo controlled trial of magnesium citrate in the treatment of chronic persistent leg cramps. Med Sci Monit 2002; 8(5):32630.

5. Woodfield R, Goodyear F, Arroll B. N-of-1 trials of quinina efficacy in skeletal muscle cramps of the leg. Br J Gen Pract 2005; 55(512):181-5

6. Cruz Arnes M, Saá Requejo CM, Moreno Hernández A, Calvo Cebrián A, Hernández Alvares LF, Calderero Castellano G. Calambres nocturnos. Revista de la SEMG 2003; 57:553-60.

7. Youn G. Calambres en las piernas. Evid Clin Concisa 2006; 5:361-2.

8. Man Son Hing M, Wells G, Lau A. Quinine for nocturnal leg cramps. A meta-analysis including unpublished data. J Gen Inter Med 1998; 13:600-6.

9. Anónimo. Trimetazidina. Disponible en: http://www.iqb.es/cbasicas/farma/farma04/t062.htm.

10. Rodríguez Padial I, Maicas Bellido C, Velázquez Martín M, Gil Polo B. Estudio prospectivo en pacientes diabéticos de la efectividad y la tolerabilidad de la trimetazidina en asociación al tratamiento previo de su enfermedad coronaria. Estudio DIETRIC. Rev Clin Esp 2005; 205(2):57-62.

11. McClellan KJ, Plosker GL. Trimetazidine. A review of its use in stable angina pectoris and other coronary conditions. Drugs 1999; 58:143-57.

12. Ruiz Meana M. Trimetazidina, estrés oxidativo y daño celular durante la reperfusión miocárdica. Rev Esp Cardiol 2005; 58(8):895-7. 